\title{
Development and Elimination of Endbulb Synapses in the Chick Cochlear Nucleus
}

\author{
Tao Lu and Laurence 0 . Trussell \\ Oregon Hearing Research Center/Vollum Institute, Oregon Health \& Science University, Portland, Oregon 97239
}

The development of synaptic function was examined at auditory nerve synapses in the rostromedial region of the cochlear nucleus magnocellularis of the chick. EPSCs were studied beginning at embryonic day 12 (E12), when synaptic transmission was first observed, through E19. The amplitude of evoked EPSCs produced by AMPA receptor (AMPA-R) increased 30-fold over this age range, whereas NMDA receptor (NMDA-R)-mediated transmission peaked at E14 and then declined almost completely. At E12, $>80 \%$ of the miniature EPSCs exhibited both receptor components, and $<10 \%$ were NMDA-R only. With age, the contribution of NMDA-R to miniature EPSCs steadily declined, suggesting that NMDA-R number is gradually reduced at individual postsynaptic sites. Between E12 and E16, the number of axonal inputs to each cell reduced by half. In simultaneous recordings from adjacent neurons, synchronous EPSCs were observed that resulted from spontaneous firing of the same presynaptic fiber. The difference in amplitude of the EPSCs in the two cells was greater in E14 than E12, whereas at E16 synchronous events were no longer observed, suggesting that the weaker input was destined for elimination. The relative amplitude of the NMDA-R component, compared with the AMPA-R component, was larger for the weaker inputs. When elimination was underway, AMPA-R quantal size was much reduced for the weakest terminals. Thus, elimination of auditory nerve terminals and pruning of axonal branches is preceded by a reduction in quantal efficacy.

Key words: auditory; AMPA; NMDA; synapse elimination; development

\section{Introduction}

Formation and refinement of glutamatergic synapses begins during embryogenesis and continues at a high rate until just after birth. Several themes appear almost universal during this time. After an initial period of excessive growth, synapses are pruned back during a process termed synapse elimination (Lichtman and Colman, 2000) and often show marked changes in the types of receptor subunits that mediate transmission (Mishina et al., 1986; Tovar and Westbrook, 1999; Cathala et al., 2000; Kumar et al., 2002). Analysis of these developmental events is complicated by the large number of inputs that single cells receive, the diverse positions of synapses on the dendritic tree, the varied sources of inputs, and the different timescales over which different types of input lay down their synapses. As a result, synaptogenesis and elimination is not well described for single axonal inputs, with respect to both the synapses they make on a given cell and the contacts a given branched axon makes on different cells.

The giant synapses between auditory nerve and neurons of the chick nucleus magnocellularis (NM) have been a valuable preparation for analysis of neuronal differentiation and survival, as well as synapse formation and elimination (Rubel and Parks, 1988). Auditory nerve axons are found in NM by embryonic day

Received Nov. 9, 2006; revised Dec. 15, 2006; accepted Dec. 18, 2006

This work was supported by National Institutes of Health Grant DC02004. We thank Dr. Trillium Blackmer for comments.

Correspondence should be addressed to Laurence 0 . Trussell, Oregon Hearing Research Center/Vollum Institute, Mail Code L-335A, 3181 SW Sam Jackson Park Avenue, Portland, OR 97239. E-mail: trussell@ohsu.edu. DOI:10.1523/JNEUROSCI.4871-06.2007

Copyright $\odot 2007$ Society for Neuroscience $\quad$ 0270-6474/07/270808-10\$15.00/0
10 (E10), and the first signs of synapse formation are seen at E11-E12 (Saunders et al., 1973; Jackson et al., 1982; Jhaveri and Morest, 1982a,b). Neurons in NM undergo a dramatic pruning of dendrites and axonal inputs, resulting by E16 in two to three large endbulb terminals on each nearly adendritic cell body in a one axon-to-one NM cell ratio (Parks, 1981; Jackson and Parks, 1982; Jhaveri and Morest, 1982a). By the time of hatching (E21), the morphological maturation is nearly complete, and the endbulbs are powerful enough to drive high-fidelity, phase-locked activity (Parks, 1981; Brenowitz and Trussell, 2001). Two types of ionotropic glutamate receptor, the AMPA and NMDA receptors (AMPA-R and NMDA-R, respectively), are expressed in NM, and proportions of different subunits are altered during developmental reorganization (Zhou and Parks, 1992; Lawrence and Trussell, 2000; Sugden et al., 2002).

We monitored transmission by single auditory nerve fibers from the time chemical transmission can be first recorded (E12), contrasting the relative contributions of AMPA-R and NMDA-R, through the period of synapse elimination and formation of mature endbulbs. Our focus was on the high best-frequency region of NM, representing cells with the earliest maturation and highest physiological demands for phase locking to acoustic input (Jackson et al., 1982). The results indicate that throughout the period of synaptogenesis and pruning, NMDA-R and AMPA-R were differentially regulated. At E12, these receptors were coactivated at most synapses, but by E19, NMDA-R were nearly absent. By recording from pairs of coinnervated neurons just before axon branches were pruned, we found that very weak synapses, which were most likely to be eliminated, had a higher proportion of NMDA-R, and a sharply reduced AMPA-R quantal size. The 
strength of the remaining synapses varied primarily in quantal content rather than quantal size.

\section{Materials and Methods}

Slice preparations and retrograde labeling of NM. The $250-\mu \mathrm{m}$-thick brainstem slices were prepared from chick embryos aged 11-19 d (E11E19) according to the protocols described previously (Zhang and Trussell, 1994). The oxygenated and warm $\left(30^{\circ} \mathrm{C}\right)$ saline for dissecting and incubating the slices contained the following (in $\mathrm{mM}$ ): $143 \mathrm{NaCl}, 5$ $\mathrm{KCl}, 3 \mathrm{CaCl}_{2}, 1 \mathrm{MgCl}_{2}, 1.8 \mathrm{Na}$-pyruvate, $1 \mathrm{NaH}_{2} \mathrm{PO}_{4}, 10 \mathrm{HEPES}, 22$ D-glucose, $\mathrm{pH}$ adjusted to 7.4 with $\mathrm{NaOH}$. To assist in the positive identification of NM neurons in slices from E12 or younger embryos, a retrograde labeling protocol described previously (Lawrence and Trussell, 2000) was adapted. First, 3-10 kDa tetramethylrhodamine dextran was dissolved in $0.1 \mathrm{M} \mathrm{NaH}_{2} \mathrm{PO}_{4}$ and allowed to dry completely. During the dissection, the freshly blocked brainstem was glued to the slicing platform; a particle of the dextran-phosphate crystal was then inserted into the tissue at midline directly ventral to the fourth ventricle, at the dorsal acoustic commissure. The brainstem was then submerged in oxygenated saline at room temperature for $1 \mathrm{~h}$ before being sliced as usual. Approximately one-half of the principal neurons in NM were positively labeled and visible under the fluorescent microscope. After a series of early experiments using the retrograde labeling, we achieved a high degree of consistency in identifying NM neurons, and the labeling was only conducted sporadically later.

Electrophysiological recordings. Whole-cell patch recordings were made with an Axopatch 200A (Molecular Devices, Sunnyvale, CA); an Axopatch $200 \mathrm{~B}$ was also used when dual recordings were needed. The oxygenated saline for perfusing slices in recording chamber was the same as the dissecting saline, except that the temperature was maintained at $35 \pm$ $1{ }^{\circ} \mathrm{C}$, and the $\mathrm{Mg}^{2+}$ was normally omitted. $\mathrm{GABA}_{\mathrm{A}}$ or glycine receptors were routinely blocked with $10 \mu \mathrm{M}$ SR 95531 (Gabazine) and $0.5 \mu \mathrm{M}$ strychnine in the bath. The internal solution for filling the recording pipettes contained the following (in mM): $108 \mathrm{CsMeSO}_{3}, 5 \mathrm{CsCl}, 1$ $\mathrm{MgCl}_{2}, 15$ phosphocreatine-Tris 2,8 BAPTA-Cs $_{4}, 10$ HEPES, 3 QX314.Cl, 4 MgATP, 0.4 Tris $_{2}$ GTP, $\mathrm{pH}$ adjusted to 7.3 at $35^{\circ} \mathrm{C}$ with TrisOH. On the day of experiments, $50 \mathrm{U} / \mathrm{ml}$ creatine phosphokinase was added from freshly thawed stock solution. The recording pipettes, pulled from thick-wall borosilicate glasses (World Precision Instruments, Sarasota, $\mathrm{FL}$ ), typically gave a series resistance of $4-10 \mathrm{M} \Omega$ before $80 \%$ compensation. A junction potential of $\sim 4 \mathrm{mV}$ was corrected for during voltageclamp experiments. Evoked EPSCs were locally elicited through a bipolar extracellular electrode fabricated from a glass theta-pipette (Sutter Instruments, Novato, CA) with a tip diameter of $5 \mu \mathrm{m}$. Compared with single-ended stimulus electrodes, with a return current path in the bath, the bipolar electrode permitted higher current density and more effective local stimulation and reduced the risk of losing recordings from strong electrical stimulation. Once the optimal location for stimulation was found, typically within $100 \mu \mathrm{m}$ of the cell, the stimulation voltage was set to $30-90 \mathrm{~V}$, while the stimulation duration was varied systematically between 10 and $250 \mu \mathrm{s}$ at an interval of $10 \mu \mathrm{s}$, as specified in acquisition software (see below), to recruit every and all excitatory inputs. Miniature EPSCs (mEPSCs) were recorded with $1 \mu \mathrm{M}$ tetrodotoxin in the bath. Although young NM neurons have dendrites, we believe these did not compromise the control of membrane voltage. mEPSC $10-90 \%$ rise times were quite rapid, averaging $167 \pm 22 \mu$ s $(n=4$ cells, E12), $162 \pm 43$ $\mu \mathrm{s}(n=6, \mathrm{E} 13-14)$, and $111 \pm 16 \mu \mathrm{s}(n=4, \mathrm{E} 16)$. Moreover, amplitude/ rise-time plots showed no correlation at any age (mean correlation coefficient $0.08 \pm 0.06, n=13$ ).

Data analyses. The data were filtered at $10 \mathrm{kHz}$, digitized with Digidata 1200 and acquired through pClamp 9 (Molecular Devices). Counting the number of inputs was usually done by counting the number of discrete steps in EPSC amplitude with increasing stimulation durations. Occasionally, the EPSC amplitude measurements were analyzed with a cluster analysis program, KlustaWin 3.1 [W. J. Heitler (University of St. Andrews, St. Andrews, UK), algorithm by K. D. Harris (Rutgers University, Newark, NJ)] for objective, unsupervised classification, and the number of classes with the best scores corroborated the manual count. Most off-line analyses were performed in Axograph 4 (Molecular Devices).
The mEPSCs containing a fast-rising AMPA component were detected using the derivative-threshold method, whereas those without a significant AMPA component were detected using a moving template whose decay kinetics resembled that of a typical NMDA-R-mediated mEPSC (Clements and Bekkers, 1997). To avoid missing a significant number of miniature EPSCs, the detection threshold was set relatively low so that $\sim 20 \%$ of the detected events were false positive and were later removed manually. NMDA-R-mediated events were also detected by eye from long periods of recordings and aligned along their rising phases. In all cases tested, these slow, irregularly rising events were eliminated by APV. To further classify mEPSCs, the ratio of areas under each event for $0-1$ to $0-10 \mathrm{~ms}$ after onset was determined. The events were then classified as "AMPA-only" if the ratio $>0.5$ and "NMDA-only" if the ratio $<0.1$; the remainder were considered "mixed."

Student's $t$ tests were used unless otherwise indicated [freeware version of KyPlot from Koichi Yoshioka (KyensLab, Tokyo, Japan)]. All of the data in the text and figures are shown as mean \pm SEM, unless specified otherwise. In the figures, ${ }^{*} p<0.05,{ }^{* *} p<0.01$, and ${ }^{* *} p<0.001$. Other analyses were performed with Origin 6.1 (OriginLab, Northampton, MA) and Igor 5.0 (Wavemetrics, Lake Oswego, OR).

Reagents. APV, 2,3-dihydroxy-6-nitro-7-sulfonyl-benzo[f]quinoxaline (NBQX), GYKI 52466 [1-(4-aminophenyl)-4-methyl-7,8-methylenedioxy$5 H$-2,3-benzodiazepine hydrochloride], SR 95531, and ifenprodil were from Tocris Bioscience (Bristol, UK). Tetrodotoxin and QX-314 were from Alomone Labs (Jerusalem, Israel). All other salts and chemicals were from Sigma-Aldrich (St. Louis, MO).

\section{Results}

\section{Amplitude and time course of evoked EPSCs}

Recordings were restricted to the rostromedial region of NM, $\sim 25 \%$ of the nucleus, to avoid issues related to developmental gradients and tonotonic variation in synaptic properties. This region contains cells responding best to higher-frequency sound. We were unable to detect EPSCs in E11 animals, whereas from E12 onwards, EPSCs were reliably evoked. These had two distinct kinetic components, a fast phase blocked by antagonists of AMPA-R and a much slower phase blocked by NMDA-R antagonists (Fig. $1 A, B$ ). Generally, the decay phase of these components were fit with a sum of two exponentials; the time constants and their weighted time constant are plotted in Figure 1, $C$ and $D$, as a function of age. AMPA-R EPSCs were dominated by a fast phase of $<0.5 \mathrm{~ms}$; a slow phase of variable duration contributed $5 \pm 1 \%$ of the fit (Fig. $1 C)(n=40$ terminals) and did not vary with age. Increases with age in decay rate were seen for both fast and slow components, but these were generally small. NMDA-R EPSCs showed no change in decay rate with development, and their overall fast phase was $60 \pm 4 \%$ of the fit (Fig. 1D) $(n=53$ synapses). This relative stability of the kinetics is in contrast to the marked changes in EPSC amplitude described below.

Previous studies have emphasized that NMDA-R components of EPSCs show striking developmental regulation of kinetics, an effect accounted for by reduced expression of NR2B subunits and increase in NR2A subunits (Tovar and Westbrook, 1999; Cathala et al., 2000). Ifenprodil (10 $\mu \mathrm{M})$, a selective antagonist of NR2Bcontaining receptors, blocked NMDA-R EPSCs by nearly $60 \%$ at both E13-E14 and E18 (Fig. 2A,C). Because the composition of synaptic and extrasynaptic NMDA-R might be different, we also examined the ifenprodil sensitivity of the response to $500 \mu \mathrm{M}$ NMDA applied by pressure ejection over the entire cell body. The degree of block was identical to that of synaptic receptors (Fig. $2 B, C)$ at the two ages. Together with the uniformity of decay kinetics between E12 and E19, these data argue against developmental regulation of NMDA-R composition during development in NM.

To examine the amplitude of EPSCs with development, we 
$A_{1}$

E12

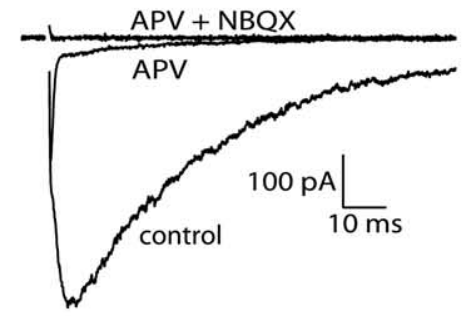

$\mathrm{A}_{2}$

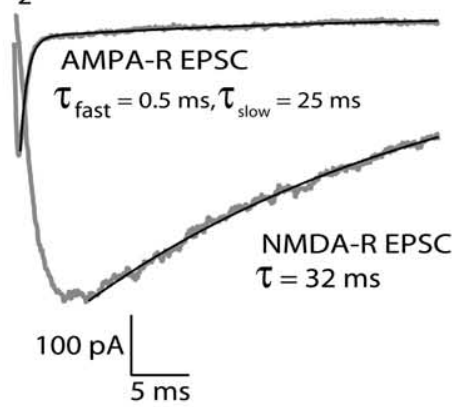

C

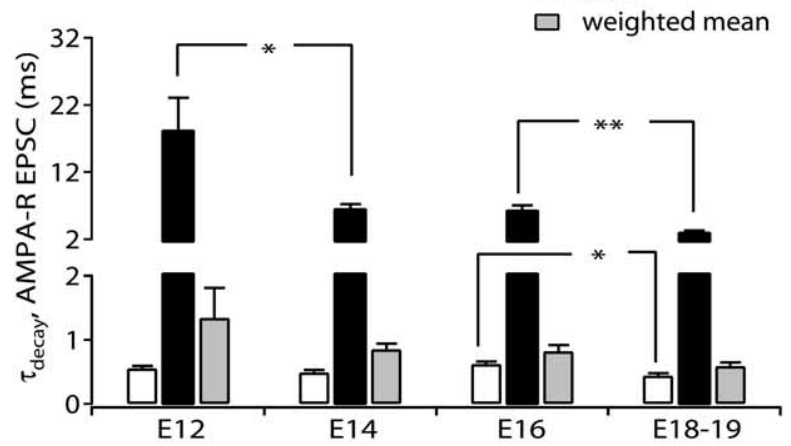

D

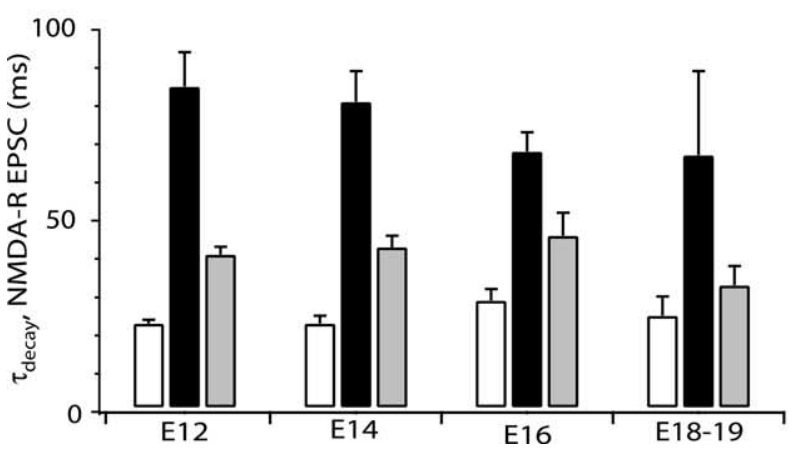

Figure 1. The time course of AMPA-R and NMDA-R EPSCs during development. $\boldsymbol{A}_{\boldsymbol{1}}$, EPSCS from an E12 NM neuron comprised AMPA-R and NMDA-R components, separable with specific antagonists, $100 \mu \mathrm{m} \mathrm{APV}$ and $20 \mu \mathrm{M} \mathrm{NBQX}$, respectively. $\boldsymbol{A}_{2}$, AMPA-R and NMDA-R EPSCs (gray lines) isolated by subtraction of current after receptor block from control records. Decays were generally best fit with the sum of two exponentials or, in a few cases such as the NMDA-R EPSC in this example, a single exponential. The exponential fits (black thin lines) are superimposed on the EPSC decay. $\boldsymbol{B}$, EPSC recorded from an E18 NM neuron was dominated by the AMPA-R component. $C$, The developmental profile of decay kinetics for AMPA-R EPSC. $n=5-9$ terminals for each age. $\boldsymbol{D}$, Decay kinetics for NMDA-REPSC during the same development period. $C, D$, Data from single-axon EPSCs (see Fig. 3). $n=7-18$ terminals for each age.
A
E13 NMDA-R EPSC

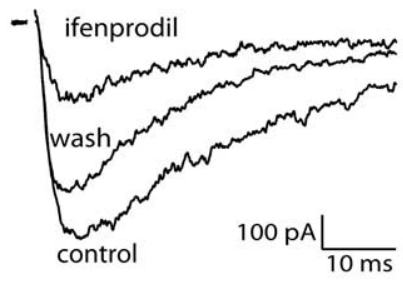

B E14, NMDA puff

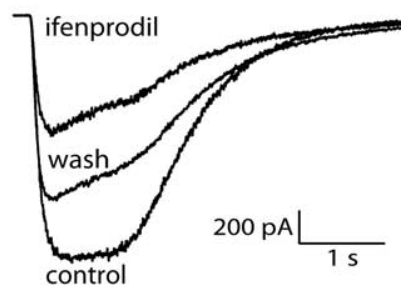

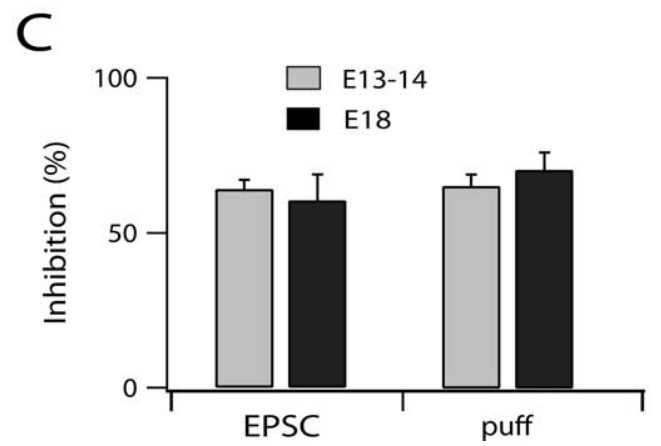

Figure 2. The sensitivity of the NMDA-R to ifenprodil remained unchanged during development. $\boldsymbol{A}$, Ifenprodil ( $10 \mu \mathrm{M}$ ) reversibly blocked $\sim 60 \%$ of the NMDA-R EPSC. The synaptic response was evoked with electrical stimulation; each trace is the average of three to five sweeps. $\boldsymbol{B}$, Ifendprodil $(10 \mu \mathrm{m})$ reversibly blocked about half of the whole-cell response to NMDA puff application (500 $\mu \mathrm{M}, 1 \mathrm{~s}$ ) in another E14 neuron. C, The bar graph summarizes ifenprodil inhibition of NMDA-R EPSC ( $n=3-6$ cells) and whole-cell response ( $n=3-13$ cells) at different ages. No significant difference was detected between the two age groups or between the two types of response mediated by NMDA-R.

wished to focus on the responses to activation of single presynaptic axons. Graded increase in either the amplitude or duration of the stimulus resulted in a clear step-like increase in the amplitude of EPSCs (supplemental Fig. 1, available at www.jneurosci. org as supplemental material), as reported by Jackson and Parks (1982); the number of such increments showed a significant decline between E12 and E16. To examine the amplitude of singleaxonal EPSCs, we used a subtractive procedure in which the mean of EPSCs of a given amplitude were subtracted from the mean amplitude after recruitment of the next input. Examples of EPSCs at different ages are shown in Figure $3 A$. The peak of the initial fast phase was taken as the AMPA-R component, whereas the amplitude 5-10 ms after onset was taken as the NMDA-R component. The mean of these amplitudes at each age is plotted in Figure 3B. Between ages E12 and E19, the AMPA-R EPSC increased over 30 -fold. The NMDA-R component initially increased at a similar rate, but by E19 it declined to one-tenth the value at E14. Thus, the ratio of the NMDA-R to AMPA-R amplitudes started out close to one at E12 and declined to near zero by E19 (Fig. 3C). This decline in NMDA-R components is consistent with the results of Zhou and Parks (1992), who found that the ability of exogenous NMDA-R to inhibit auditory nerve transmission declined during embryogenesis. In some experiments, we examined the amplitude of EPSCs at the caudolateral end of $\mathrm{NM}$, which is reported to mature later and have smaller EPSCs (Jackson et al., 1982; Fukui and Ohmori, 2004). As shown with red triangles in Figure 3, $B$ and $C$, at E19 the AMPA-R EPSCs were indeed smaller than in the rostral end; however, the NMDA-R component was larger, resulting in a larger NMDA/AMPA ratio. 
A
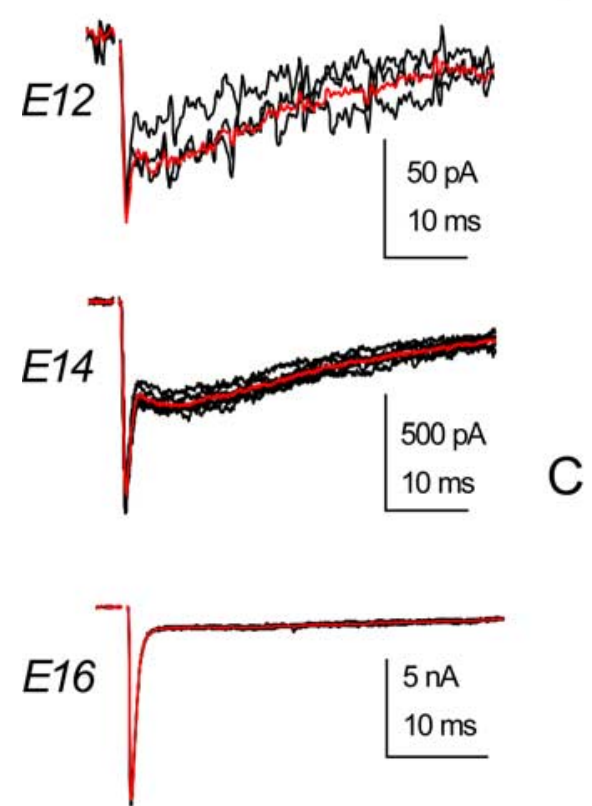

B

C
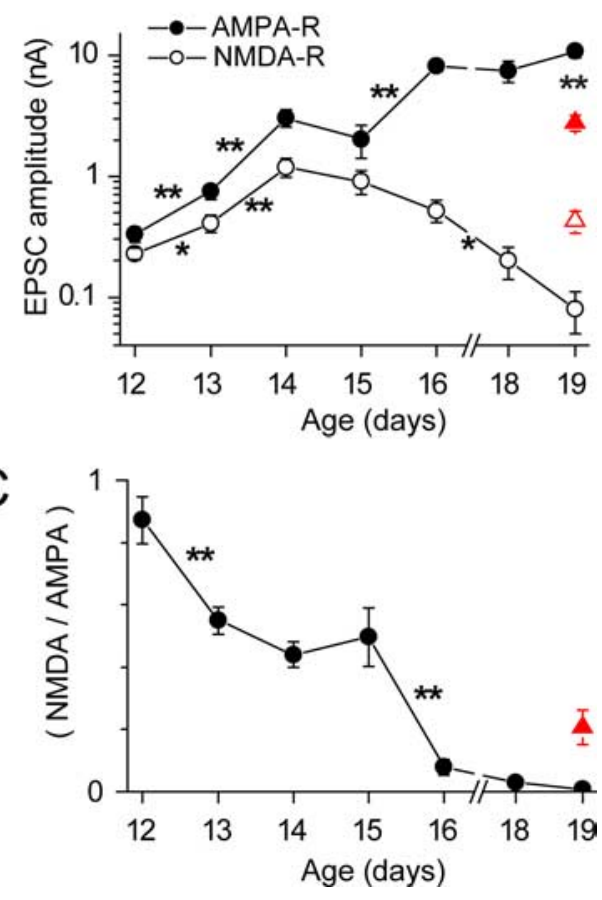

these three ages. At E12, most events spanned the range from 100 to $600 \mathrm{pA}$. By E16, although the mean amplitude was much larger, the log range was similar, from 1 to $8 \mathrm{nA}$. However, at E14, the EPSCs clearly comprised two classes, one spanning a range similar to that seen in E16 but another spread between $\sim 30$ and $1000 \mathrm{pA}$, similar to that seen in E12. The amplitude of NMDA-R components for the EPSCs in 4-AP also described a pattern similar to that seen in evoked events, with mean amplitudes increasing between E12 and E14 and declining thereafter (Fig. $4 E, F)$. Thus, these data support the observation that NMDA-R components and input number decline developmentally in $\mathrm{NM}$ and that, at E14, when NMDA-R components are largest, cells receive contact from terminals with widely differing synaptic strengths. It is likely therefore that most axonal inputs are laid down by E12 and that subsequent events involve either the elimination or further growth of these terminals.

Figure 3. Developmental changes in the strength of single-axon EPSCs mediated by AMPA-R or NMDA-R. A, Single-axon EPSCS recorded in neurons from different ages, with the average responses (red lines) superimposed. Note the changing relative amplitudes of the slower NMDA-R component and faster AMPA-R component. $B$, Summary of the amplitude of single-axon AMPA-R and NMDA-R EPSCs during development. The AMPA-R EPSC almost continuously grew in strength, whereas the NMDA-R EPSC displayed a bell-shaped growth curve. In neurons from the caudal end of NM (red triangles), the difference between AMPA-R and NMDA-R EPSCs was less. Note log scale. C, The relative strength of single-axon EPSCs mediated by NMDA-R or AMPA-R declined throughout the developmental period. A more modest reduction in the relative strength was seen in caudal neurons (red triangle).

It should be noted that, in our experiments, no evoked EPSC at E12 lacked an AMPA-R component.

\section{Spontaneous EPSCs triggered in 4-AP}

Estimation of EPSC amplitude and input number by graded stimulus duration is limited by the possibility that fibers may have similar spike thresholds. To avoid this potential confound, we examined spontaneous EPSCs (sEPSCs) that occurred in the presence of 30-100 $\mu \mathrm{M}$ 4-aminopyridine (4-AP). Under these conditions, presynaptic fibers began firing at low rates (Fig. 4A). Data were recorded continuously and EPSCs were extracted and aligned by an event-detection routine using a threshold amplitude of 20 pA. sEPSCs were characterized by a flurry of delayed release events after the initial EPSC, probably an effect of 4-AP on the presynaptic $\mathrm{Ca}^{2+}$ transient. In this way, occasional, small sEPSCs could be distinguished from miniature EPSCs (see below). As seen in the right panel of Figure $4 A$, events occurred in characteristic groupings of amplitude, suggesting that we could use these groupings to estimate the number of inputs in each cell, with the limitation that inputs producing EPSCs of similar amplitude would be indistinguishable. Estimating the number of inputs in each cell by this method gave values similar to those seen with grading stimulus duration, with values of 4 inputs at E12 to 1.8 inputs at E16 (Fig. $4 D$ ). Figure $4 B$ shows the amplitudes of discrete components seen in different cells at three ages. The data show that at an intermediate age (E14), the range of amplitudes was widest; some EPSCs were as large as those seen in E16, but much smaller ones also occurred in some cells. Figure $4 C$ plots the amplitudes in terms of cumulative percentage of all events for

\section{Paired recordings}

The differences in the amplitudes of EPSCs at E14, and the decline in input number with age, suggest that smaller inputs could reflect inputs that are destined for, or in the process of, elimination. We examined this possibility by recording from adjacent cells, with the expectation that, before establishment of a one-to-one innervation ratio, branches of single axons may innervate neighboring neurons. 4-AP was used to trigger spontaneous firing. An event detection routine was used in which EPSCs from one cell triggered off-line acquisition of sweeps in both cells. In this way, we searched for EPSCs that occurred synchronously (within $1 \mathrm{~ms}$ ) in both cells. Figure $5 A$ illustrates one such recording from an E12 animal. The events marked by triangles show a fiber that produced large EPSCs in cell 2 and tiny EPSCs in cell 1, whereas squares mark EPSCs of comparable level, which we presume was elicited by spikes in another fiber. We interpret these results as indicating that at least two axons contacted the same pair of neurons, because synchronous EPSCs occurred repeatedly with characteristic size and shape ( $n=18$ for EPSC pairs marked by triangle and $n=9$ for EPSC pairs marked by the square). Figure $5 B$ shows a recording from an E14 embryo in which very large EPSCs in cell 1 were matched to much smaller events in cell 2. In an E16 pair (Fig. 5C), synchronous EPSCs were not found, which suggests that these cells did not share inputs. The convergence of these events was also examined by constructing cross-correlations of activity in the two sets of traces; these showed peaks consistent with the by-eye observations of synchronous events in the eventdetection routine (data not shown).

The number of apparent dual innervations and the chance of finding them during development provided another index of synapse elimination. Using the shape and amplitude of EPSCs to identify distinct inputs, we plotted the number of paired connections (i.e., the number of branched axons ending on the same pair of cells) at E12, E14, and E16. This number declined from $\sim 2.5$ at 

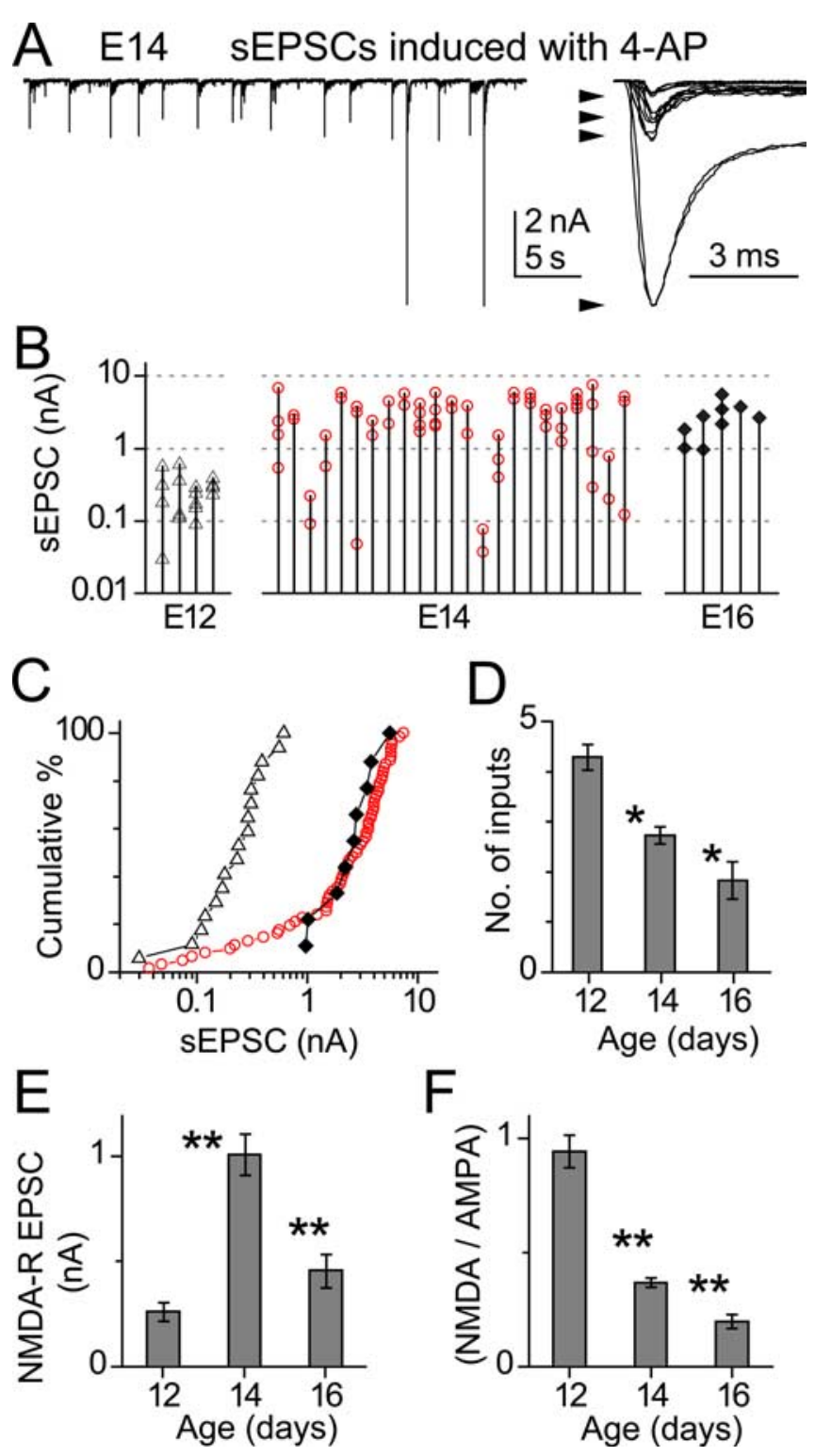

Figure 4. Single-axon EPSCs induced with 4-AP. A, Left, Spontaneous EPSCS (SEPSCS) in an E14 neuron after bath application of $30 \mu \mathrm{m} 4-\mathrm{AP}$. Right, sEPSCs from the same cell overlaid. Four distinct amplitude groups can be discerned corresponding to four inputs. B, sEPSC amplitudes seen in each cell at three ages. For each cell, mean amplitudes in each size group are connected by a vertical line. Thus, the number of points along each line is the number of inputs. Note log scale. $\boldsymbol{C}$, Cumulative percentage plot of the data from $\boldsymbol{B}$, preserving the grouping according to age ( $\triangle$ for E12, red $\bigcirc$ for E14, and $\checkmark$ for E16). D, Summary of the estimated number of inputs at various ages. Significant decrease was detected between the consecutive age groups. $\boldsymbol{E}$, The average amplitude of NMDA-R component in the sEPSCs changed with age. The development of NMDA-R component was bell-shaped, peaking at E14. $\boldsymbol{F}$, The relative strength of NMDA-R over AMPA-R component of the sEPSCs declined continuously during development.

E12 to zero at E16 (Fig. 5D). Similarly, the success rate of finding pairs of cells with common innervation also declined, from $100 \%$ at E12 to zero at E16 (Fig. 5E). These data are consistent with those of Figure 4 and supplemental Figure 1 (available at www. jneurosci.org as supplemental material), showing a 50\% decline in the number of inputs per cell, and suggest that overall loss of synaptic inputs to each cell is attributable to pruning of axonal branches. The relative amplitude of the AMPA-R components of the two EPSCs produced by single axons in the pair varied widely (Fig. $5 F$ ) and could be nearly identical or differ by a factor of 40 . At E14, the relative amplitude of NMDA-R and AMPA-R com- ponents revealed that the weaker of the two inputs had larger NMDA-R/AMPA-R ratios (Fig. 5G). This observation for inputs of the same developmental age reinforces the earlier observation that NMDA-R component declines as AMPA-R components grow between ages E14 and E19 (Fig. 3B).

\section{Receptor composition in mEPSCs}

To track the contribution of AMPA-R and NMDA-R over development at single synaptic sites, we recorded mEPSCs in the presence of TTX. Figure $6 A$ shows continuous traces in control and APV-containing solutions; APV eliminated small, slow tails on the decay phase of a portion of the mEPSCs, whereas NBQX blocked all fast rising and decaying events (data not shown). Figure $6 B$ shows events in control solutions in the same E14 cell, categorized by the magnitude of the initial and late components. Events were sorted into three classes, AMPA only, NMDA only, and mixed (see Materials and Methods). Between E12 and E18, the overall mEPSC frequency increased approximately fivefold, a smaller increase than that of the peak amplitude of the evoked EPSC (Fig. 6C). The average peak amplitude of mEPSCs mediated by AMPA-R changed little over this time period, increasing from $60 \mathrm{pA}$ at E12 to $105 \mathrm{pA}$ at E18 (Fig. 6D). The amplitude of NMDA-R components of mixed or NMDA-only mEPSCs described a progression somewhat different from the amplitude of the NMDA-R component of evoked EPSCs (Fig. 3B). Between E12 and E14, the mEPSCs mediated by NMDA-R hovered at $\sim 30$ $\mathrm{pA}$, with no significant change in amplitude, whereas by E15 and E16, the amplitude dropped suddenly to a few picoamperes (Fig. $6 E)$. Looking at the relative fraction of all events in the three categories, we observed a steady decline in the proportion of mixed events with age. NMDA-only and AMPA-only events each comprised only $\sim 10 \%$ of events at E12. The former remained rare, eventually disappearing after a few days, whereas the latter increased to $100 \%$ of all events by E18. These data suggest that, initially, NMDA-R and AMPA-R share most individual synaptic sites and that the density of NMDA-R at each site gradually declines with development.

\section{Regulation of quantal size}

The data in Figure 6D indicate that the average peak amplitude of mEPSCs changed modestly during development, despite enormous changes in the amplitude of the evoked EPSC. However, we also showed that at any one age, and particularly near E14, the sizes of different inputs to single cells can vary widely; such variation might obscure input-specific difference in quantal properties. We therefore asked if quantal size might vary with input strength, by comparing the size of the spike-triggered EPSC to that of the mean of delayed-release events that follow the EPSC. Delayed release events were taken to reflect the quantal size for that terminal (Bekkers and Clements, 1999). Figure 7A shows synchronous EPSCs in a paired recording; to the right are the delayed events extracted with a template-matching routine. The sEPSCs differed by 17 -fold in amplitude, and the quantal currents associated with the stronger inputs were twofold larger than those of the weaker input. To confirm that these differences were apparent for the AMPA-R component alone and did not reflect differences in the summation of AMPA-R and NMDA-R components, the experiment was repeated in APV, and similar results were obtained (Fig. 7A, red traces). Indeed, because APV had little effect on the time course of delayed events, these quantal currents were mediated primarily by AMPA-R. Data like these for the intermediate ages E13-E15 are summarized in Figures $7 C-E$. Figure $7 C$ shows the quantal size plotted against the peak EPSC 

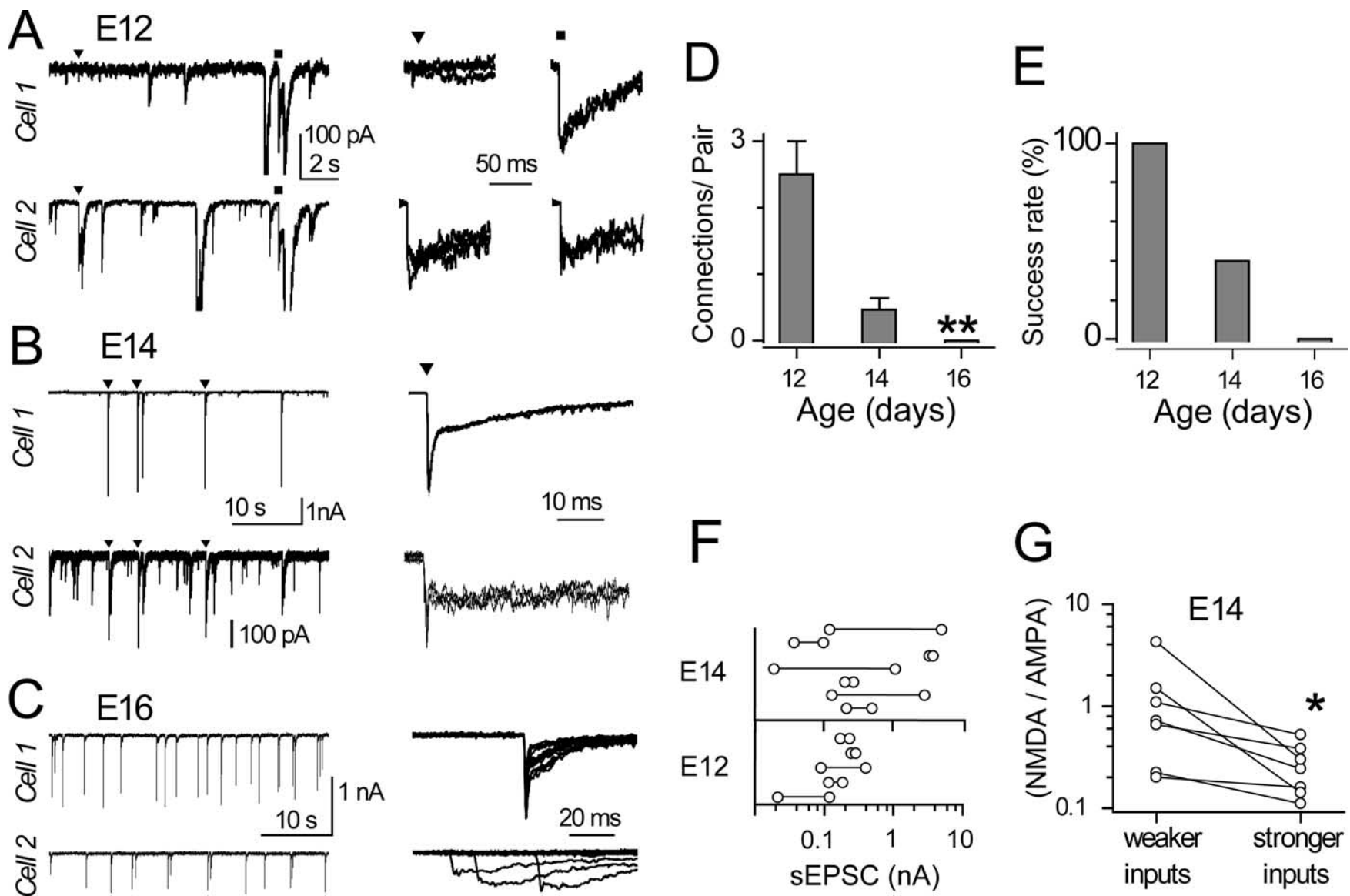

Figure 5. Dual recordings of spontaneous EPSCS. A, Top and bottom traces in the left panel show spontaneous EPSCs simultaneously recorded from adjacent E12 neurons, in the presence of 30 $\mu \mathrm{m}$ 4-AP. Pairs of synchronous events with similar shape or relative amplitudes were presumed to be generated by the same axon. Examples from two such axonal inputs, labeled $\boldsymbol{\nabla}$ and $\boldsymbol{\square}$, are overlaid and displayed in the right panel. For the pair on the left $(\boldsymbol{\nabla})$, the sEPSC in cell 1 is tiny when compared with that in cell 2 , whereas for the pair on the right $(\boldsymbol{\square})$, both sEPSCs are comparable in size. $\boldsymbol{B}$, Similar dual recording from adjacent E14 neurons revealed only one pair of synchronous sEPSCs. Note the vertical scale differs by tenfold between the top and bottom traces. $\boldsymbol{C}$, Another dual recording from adjacent E16 neurons gave no synchronous SEPSCs, as demonstrated on the right by the random misalignment of sEPSCs in bottom traces when the sEPSC s in top traces were aligned. $D$, The average number of instances of paired connections found in all of the dual recordings made at various ages. A significant decrease was detected, consistent with the reduction in axon bifurcation during development. $\boldsymbol{E}$, The success rate of finding any common innervation for all of the dual recordings at a given age. The number of dual recordings were 3 for $E 12$, 16 for $E 14$, and 4 for E16. F, Peak AMPA-R amplitude of sEPSCS, with the horizontal line connecting EPSCs produced by the same axon in adjacent neurons. G, At E14, the NMDA-R component is significantly more prominent in the weaker sEPSCs than in the stronger ones when each two synchronous sEPSCs were compared side-by-side.

size for E13-E15 embryos (black symbols); Figure 7D is an expanded version showing the smallest events. The data reveal an initial fast increase in quantal size with sEPSC followed by shallower linear phase. Thus, although in general smaller sEPSCs had smaller quantal size, this relationship was particularly sharp for the very weakest sEPSCs. Given the ages represented by these data, it is likely that these weakest EPSCs were generated by terminals that were nearly eliminated. Is their smaller quantal size a special feature of eliminating synapses, or instead a feature of any immature terminal? The data in green in Figure 7, $C$ and $D$, are from five terminals taken from E12 embryos. These generated sEPSCs of amplitude comparable with the weakest inputs in the older embryos but with a larger quantal size. Indeed, for E13-E15 sEPSCs $<400 \mathrm{pA}$, the mean quantal size was $36 \pm 6 \mathrm{pA}$, whereas for the E12 data set, the mean quantal size was $79 \pm 9 \mathrm{pA}$ ( $p=$ 0.002 ). The mean sEPSC for the older and younger group were quite similar, at $160 \pm 40 \mathrm{pA}$ and $145 \pm 30$ (not significant), respectively. Thus, it is likely that the quantal size is reduced in a subpopulation of weak terminals during the period of elimination.

Using this estimate of quantal size, we were also able to estimate the quantal content (sEPSC/quantal current) for E13-E15 embryos and found a steep, linear relation with sEPSC size (Fig. $7 E)$. E12 terminals also fell along this relation (green symbols) and thus showed very low quantal content; indeed, one E12 EPSC was apparently generated by a single quantum. A linear relationship was also observed between quantal size and stimulus-evoked EPSCs (Fig. $7 B, F$ ); here we did not observe very small quantal sizes, which we attribute to the low probability of stimulating extremely weak inputs in isolation. Together, these data indicate that variation in the strength of auditory nerve transmission, across development or at a single age, reflects both change in the quantal size and quantal content.

\section{Discussion}

Receptor composition at single synaptic sites

We recorded EPSCs in NM as early as E12. Saunders et al. (1973) and Jackson et al. (1982) reported field potential responses from E11 chicks; however, these recordings were apparently rare, and synapses would likely have been missed using single-cell sampling. This timing is also consistent with morphological observations, which show auditory nerve fibers proliferating in NM at E11-E13 and synaptic profiles visible at E12 (Jackson and Parks, 1982; Jackson et al., 1982). Moreover, Hendricks et al. (2006) 
described the synaptic marker SV2 in NM as "faint" at E9 but "increasing" by E12. Given the relative size of AMPA-R EPSCs and quantal current, auditory nerve terminals form fewer than six synaptic sites on each NM cell between E11 and E12. The changes that follow are remarkable. Within 1 week, one-half of the contacts are withdrawn, whereas each remaining terminal grows into one of the most powerful inputs in the brain, with hundreds of release sites and a quantal size larger than that at most central glutamatergic synapses (Trussell, 1999). Our data show that this process involves distinct phases of postsynaptic regulation.

\section{Synapse elimination and pruning of axon branches}

These results are consistent with the observations of Jackson and Parks (1982), who recorded multiple steps in EPSP amplitude and morphologically identified multiple innervation in chick, demonstrating that loss of preterminal branches paralleled the reduction of function multiple innervation. Our study is the first to monitor physiologically the strength of contacts both during development and at different branches of the same axon. By E14, we found evidence for two populations of terminal on each cell: one similar in strength to that seen at E16, and another closer in strength to the weaker terminals of E12 (Fig. 4). It seems reasonable that, between these two populations, it is the weaker synapses that will be eliminated. An alternative possibility is that the weakest inputs at E13-E15 have only just formed; this is made less likely by the steady progression in synapse elimination from E12 (i.e., inputs are only lost, not added) and the differences in quantal properties discussed below. Given that this period of development is also marked by almost total dendritic removal, it may be that many or most eliminating terminals are found on dendrites and not cell bodies.

Our study suggests that terminals on the verge of elimination undergo a sharp reduction in quantal size. Although a positive relationship between quantal size and the EPSC was found for all synapses between E12 and E15, this correlation was steepest for the weakest terminals during the period of elimination. Terminals from younger embryos were also weak but did not feature such a reduced quantal size. This change in quantal size could reflect differences in either the number of AMPA-R, the properties of those receptors, or the glutamate content of vesicles. The more gradual increase in quantal size across the whole population could reflect changes in AMPA-R subunit composition. Lawrence and Trussell (2000) showed that AMPA-R in NM undergo a switch in properties

A

B

E
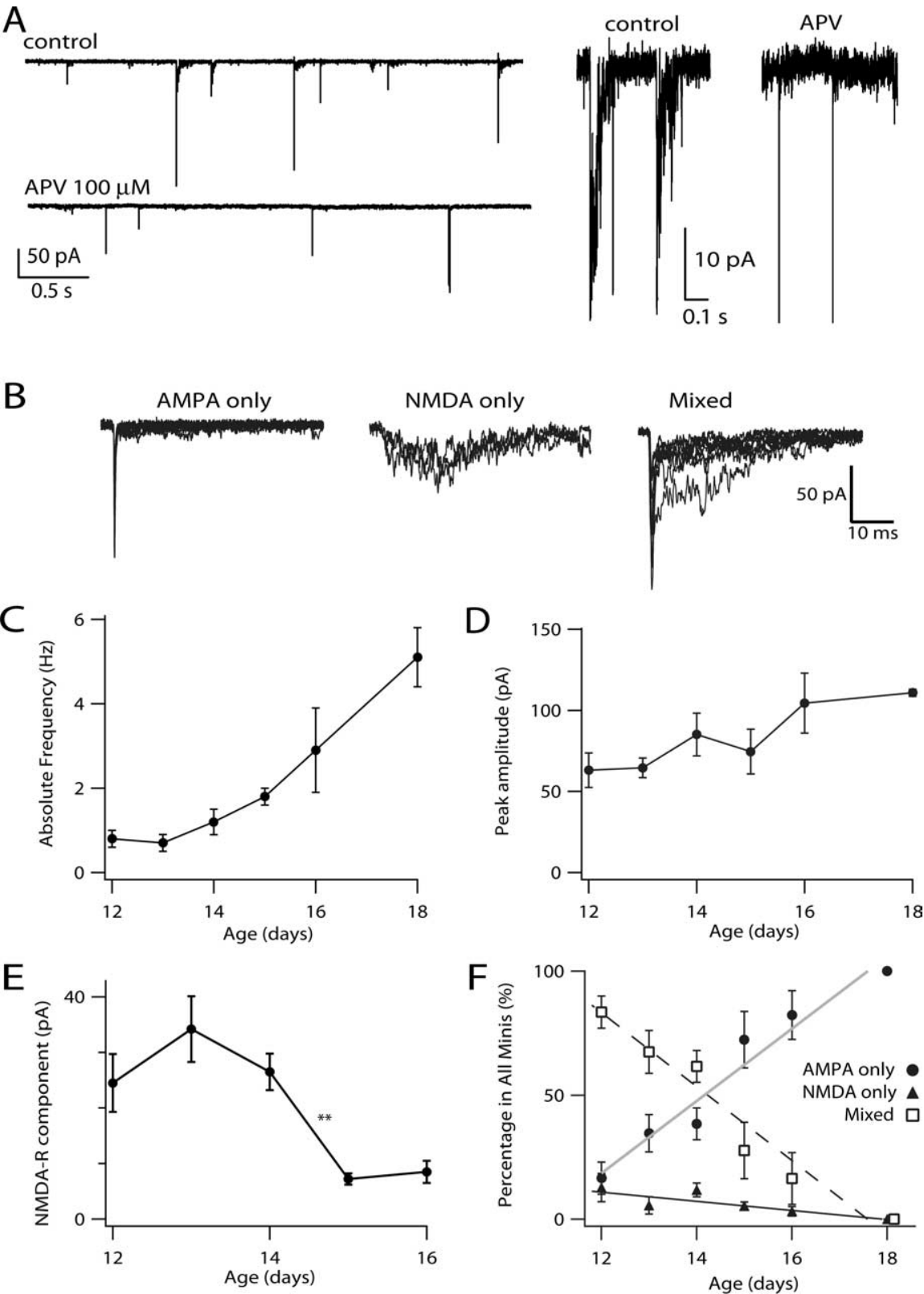

Figure 6. mEPSCs showed varied contribution from NMDA-R and AMPA-R. $\boldsymbol{A}$, Examples of mEPSCs recorded in the one E14 neuron in the presence of $1 \mu \mathrm{m}$ tetrodotoxin. The addition of $100 \mu \mathrm{M}$ APV helped confirm the existence of predominantly AMPA-only mEPSCs, NMDA-only mEPSCs, or those with mixed contribution from AMPA-R and NMDA-R ("Mixed"). Right, Portions of the recording on the left on an expanded time scale, highlighting the apparent effect of APV. $\boldsymbol{B}$, From the same neuron in $\boldsymbol{A}$, overlaid mEPSCs classified as AMPA only, NMDA only, or mixed. C, Average frequency of mEPSCs shows a fivefold increase during development ( $p=0.02$, ANOVA), consistent with an increase in the number of release sites or release probability. $D$, The average peak amplitude of mEPSCS showed only a modest (less than twofold) but significant increase during development ( $p=0.0002$, ANOVA). $\boldsymbol{E}$, The average amplitude of NMDA-R component (excluding AMPA only events) decreased significantly between E14 and $\mathrm{E} 15(p=0.005, \mathrm{ANOVA})$, consistent with the removal of NMDA-R from postsynaptic sites. $\boldsymbol{F}$, The relative fraction of all events in the three categories and linear fits. During development, the relative fraction of AMPA-only or mixed events changed significantly $(p=0.0002$, ANOVA), whereas NMDA-only mEPSCs remained rare $(p>0.05$, ANOVA).

during development, resulting in faster desensitization and less association with the glutamate receptor 2 (GluR2) subunit. Desensitization, though not playing a role in the mEPSC decay, could account in part for the slight speeding of the evoked EPSC (Otis et al., 1996). GluR2 is associated with lower channel conductance, and thus a reduction in GluR2 itself would be expected to lead to larger quanta (Swanson et al., 1997). For the weak, eliminating synapses, it is probable that reduction in AMPA-R 

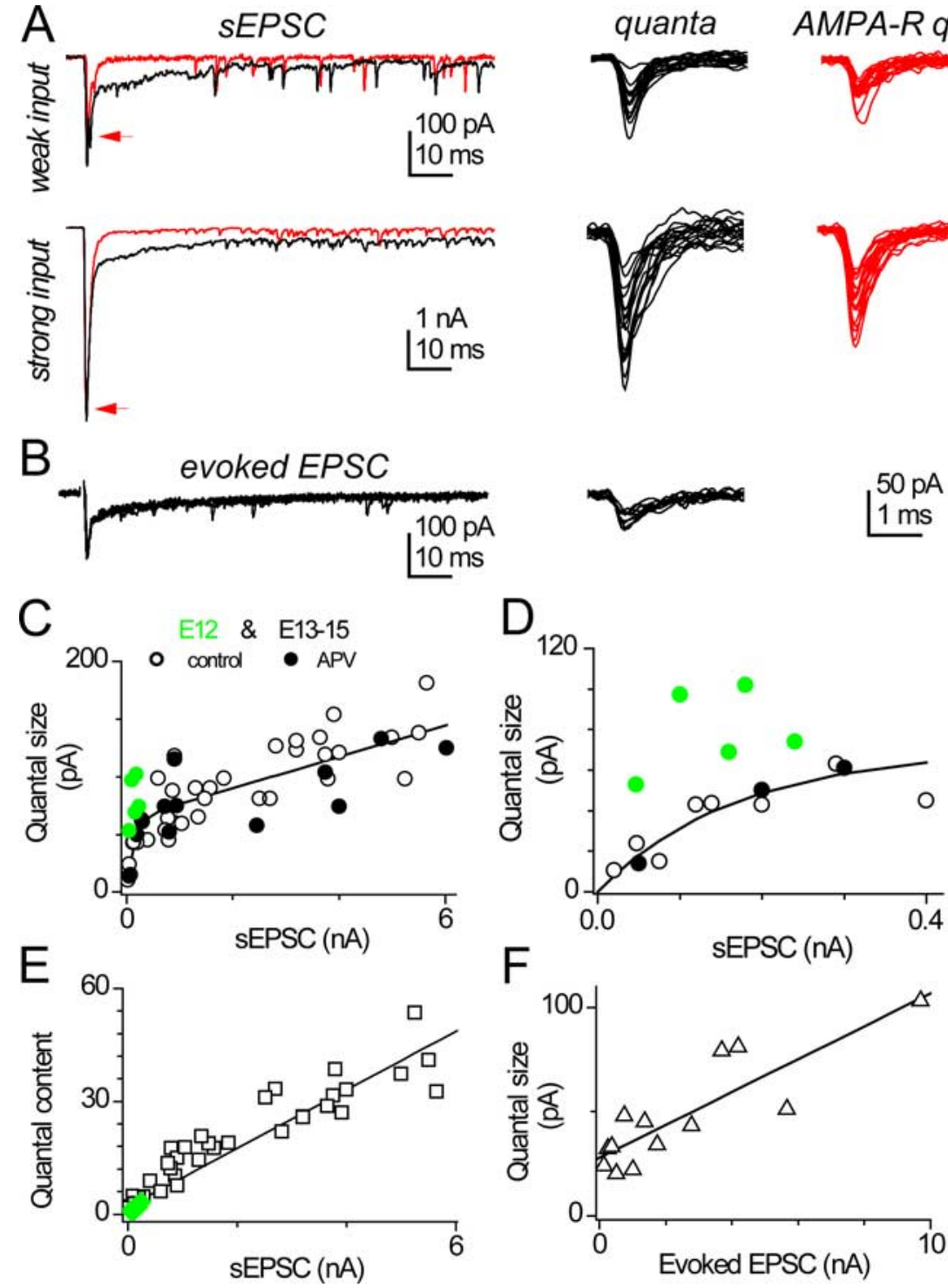

Figure 7. Delayed releases after spike-triggered EPSCs at intermediate age. $\boldsymbol{A}$, Left, Spontaneous EPSCs(sEPSCs) and numerous delayed-release events in the presence of $50 \mu \mathrm{m}$ 4-AP. Data in this example are from paired recordings in adjacent E14 neurons. Note the tenfold difference in vertical scales. The addition of $100 \mu \mathrm{M}$ DL-APV blocked all NMDA-R, which had only minor contribution to the peak response (red line). Right, Overlaid delayed releases collected from the tails of sEPSCs, with (red) or without APV, and the smaller delayed releases were preceded by the weaker SEPSC. $\boldsymbol{B}$, In an alternative protocol, delayed releases were collected in another E14 neuron after the electrically evoked EPSCs, in the presence of $3 \mathrm{~mm} \mathrm{SrCl}_{2}$. C, The quantal size, as measured with the average peak amplitude of delayed releases, shows strong positive correlation to the amplitude of sEPSC for data from E13-E15 chicks (black symbols). The fitted line was of the following form: quantal size $=(63 \mathrm{pA}+0.014 \times \mathrm{EPSC}) \times(1-$ $\exp (-E P S C / 134 p A))(r=0.79, p<0.0001)$. This curve has an exponential rise followed by a linear slope. Data were pooled from 19 neurons aged E13-E15, with each neuron contributing data at $1-4$ distinct SEPSC amplitudes. Open symbols are recordings in control solutions, whereas filled symbols are from APV-containing solutions to block NMDA-R. Green symbols are data from five E12 synapses. D, Same as $\boldsymbol{C}$ but different scale. $\boldsymbol{E}$, the quantal content, the ratio of sEPSC amplitude over average quantal size, is linearly correlated with the amplitude of sEPSC $(r=0.93, p<0.0001)$. Black symbols are P13-P15 cells, whereas green symbols are from E12. $\boldsymbol{F}$, The correlation also appears between the average quantal size and the amplitude of stimulus evoked EPSC recorded in $3 \mathrm{~mm} \mathrm{SrCl}_{2}$ plus $3 \mathrm{~mm} \mathrm{CaCl}(0.86, p=0.0002)$, indicating that it is not an artifact related to the 4-AP used in triggering sEPSCs. Data were pooled from seven neurons aged E14. When multiple axons were stimulated, averaged amplitude of EPSC was used.

number or glutamate content of vesicles underlies the reduced quantal size. If the latter, however, it is clear that a reduced availability of glutamate or vesicular glutamate transporters would have to be bouton specific, because the terminal on the other, noneliminating branch of the same axon generated a normal quantal size. We therefore favor the hypothesis that withdrawal of terminals is preceded by removal of AMPA receptors. The rela-
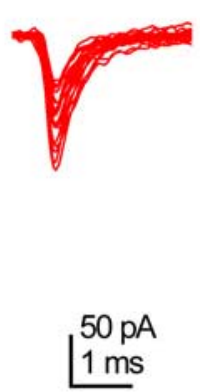

tively higher proportion of NMDA-R at the weaker inputs (Fig. 5G) would be consistent with this hypothesis.

Physiological analysis of synapse elimination has been performed for several other CNS regions (Chen and Regehr, 2000; Hashimoto and Kano, 2003; Kim and Kandler, 2003). Chen and Regehr (2000) observed an increase in quantal size with age but did not distinguish between different classes of terminal at a given age. Hashimoto and Kano (2003) were able to differentiate between climbing fiber terminals that were most likely to be eliminated based on their strength. They found that these exhibited a reduced glutamate transient attributable to less multivesicular release and reduced release probability. However, in their study, the quantal size, determined as delayed release events after replacement of bath $\mathrm{Ca}^{2+}$ with $\mathrm{Sr}^{2+}$, was not different for weak and strong inputs. It is possible that the weakest terminals were not functional in the presence of $\mathrm{Sr}^{2+}$. Rather, our results are consistent with the events underlying synapse elimination at the neuromuscular junction (Rich and Lichtman, 1989; Balice-Gordon and Lichtman, 1993; Colman et al., 1997), in which quantal size and content both decline before withdrawal of axon branches. Thus, elimination at peripheral and central synapses may employ similar mechanisms.

Development of giant terminals has also been examined in the mammalian auditory system. At the calyx of Held synapse in mammals, functional innervation has been documented as early as postnatal day 1 (Hoffpauir et al., 2006), 10 d before hearing onset. Unlike in chick NM, postsynaptic medial nucleus of the trapezoid body (MNTB) neurons receive only one calyx, although branchlets from the calyx may contact neighboring cells (Hoffpauir et al., 2006; Rodriguez-Contreras et al., 2006). Presynaptic axons, however, do branch to produce calyces on several nearby cells (Rodriguez-Contreras et al., 2006). In NM, such branches were common at early stages of synaptogenesis, and their pruning appears to be the basis of synapse elimination. Similar information is not available for mammalian endbulb development, specifically for the large terminals on spherical bushy cells. However, given that a small number of endbulbs, perhaps one to three (Nicol and Walmsley, 2002), contacts each spherical bushy cell, it may be that pruning takes place there in a similar manner.

\section{Function and regulation of NMDA receptors}

Before completion of synapse elimination, the chances that an axon sends branches to nearby NM cells is relatively high. One of 
these branches is destined to be eliminated, and indeed we observed that the strength of contacts made by each branch could differ widely, particularly at E14, when synapse elimination is imminent. Moreover, the smaller inputs at a given age tended to have a higher contribution of NMDA-R to their synaptic response. Overall, NMDA-R content peaked at about the time that synapse elimination was nearly complete. These results are consistent with a model in which transmission by either very young or dying terminals is dominated by NMDA-R. One interpretation of this is that synaptic weakening and loss is triggered by excessive NMDA-R activity (Rabacchi et al., 1992; Kakizawa et al., 2000). Although AMPA-R in NM are $\mathrm{Ca}^{2+}$ permeable, their much briefer open times would permit much less $\mathrm{Ca}^{2+}$ entry than NMDA-R (Lawrence and Trussell, 2000). Although analysis of the expression of the NR1 subunit of NMDA-R shows little developmental downregulation in NM (Tang and Carr, 2004), expression of other subunit components of the NMDA-R could be rate limiting for its expression at synapses in NM. Another possibility is that it is the failure to recruit AMPA-R that causes synaptic, or even dendritic, withdrawal (Haas et al., 2006). Genetic alteration in the expression of AMPA-R or NMDA-R subunits could provide a tool to test these hypotheses.

The downturn in NMDA-R expression is similar to that seen in other large synapses, with some exceptions. For example, at mammalian endbulbs of the ventral cochlear nucleus and at the calyx of Held in the MNTB, NMDA-R EPSCs also decline during postnatal development (Bellingham et al., 1998; Taschenberger and von Gersdorff, 2000; Joshi and Wang, 2002). However, the decline there is accompanied by a change in receptor kinetics and pharmacology consistent with a switch from NR2B to NR2A subunits in the NMDA-R. For the chick, the lack of change in ifenprodil block and in the NMDA-R EPSC decay time suggests that only one combination of subunits is employed in NM development. Moreover, the speed of EPSC decay and its partial sensitivity to ifenprodil is consistent with characteristics of NR2B containing receptors at all ages that we have examined.

In our studies of evoked EPSCs at E12, we did not detect axonal inputs that only used NMDA-R, i.e., "silent synapses." Analysis of AMPA and NMDA components in mEPSCs revealed only a small population of NMDA-R-only synaptic sites. Thus, it does not appear that auditory nerve synaptogenesis in NM is initiated with formation of a silent synapse in contrast to observations made at some synapses (Durand et al., 1996; Wu et al., 1996; Isaac et al., 1997). Rather, our data indicate that auditory nerve synapses are "born" with both receptor subtypes, and the contribution of NMDA-R to each synaptic site then falls off gradually with age. As the terminal is strengthened, new sites must undoubtedly form, particularly as dendrites are removed and the endbulb coalesces. These appear either to be entirely free of NMDA-R right from the beginning or to lose them so fast that they were not detectable.

\section{References}

Balice-Gordon RJ, Lichtman JW (1993) In vivo observations of pre- and postsynaptic changes during the transition from multiple to single innervation at developing neuromuscular junctions. J Neurosci 13:834-855.

Bekkers JM, Clements JD (1999) Quantal amplitude and quantal variance of strontium-induced asynchronous EPSCs in rat dentate granule neurons. J Physiol (Lond) 516:227-248.

Bellingham MC, Lim R, Walmsley B (1998) Developmental changes in EPSC quantal size and quantal content at a central glutamatergic synapse in rat. J Physiol (Lond) 511:861-869.

Brenowitz S, Trussell LO (2001) Maturation of synaptic transmission at end-bulb synapses of the cochlear nucleus. J Neurosci 21:9487-9498.
Cathala L, Misra C, Cull-Candy S (2000) Developmental profile of the changing properties of NMDA receptors at cerebellar mossy fiber-granule cell synapses. J Neurosci 20:5899-5905.

Chen C, Regehr WG (2000) Developmental remodeling of the retinogeniculate synapse. Neuron 28:955-966.

Clements JD, Bekkers JM (1997) Detection of spontaneous synaptic events with an optimally scaled template. Biophys J 73:220-229.

Colman H, Nabekura J, Lichtman JW (1997) Alterations in synaptic strength preceding axon withdrawal. Science 275:356-361.

Durand GM, Kovalchuk Y, Konnerth A (1996) Long-term potentiation and functional synapse induction in developing hippocampus. Nature 381:71-75.

Fukui I, Ohmori H (2004) Tonotopic gradients of membrane and synaptic properties for neurons of the chicken nucleus magnocellularis. J Neurosci 24:7514-7523.

Haas K, Li J, Cline HT (2006) AMPA receptors regulate experiencedependent dendritic arbor growth in vivo. Proc Natl Acad Sci USA 103:12127-12131.

Hashimoto K, Kano M (2003) Functional differentiation of multiple climbing fiber inputs during synapse elimination in the developing cerebellum. Neuron 38:785-796.

Hendricks SJ, Rubel EW, Nishi R (2006) Formation of the avian nucleus magnocellularis from the auditory anlage. J Comp Neurol 498:433-442.

Hoffpauir BK, Grimes JL, Mathers PH, Spirou GA (2006) Synaptogenesis of the calyx of Held: rapid onset of function and one-to-one morphological innervation. J Neurosci 26:5511-5523.

Isaac JT, Crair MC, Nicoll RA, Malenka RC (1997) Silent synapses during development of thalamocortical inputs. Neuron 18:269-280.

Jackson H, Parks TN (1982) Functional synapse elimination in the developing avian cochlear nucleus with simultaneous reduction in cochlear nerve axon branching. J Neurosci 2:1736-1743.

Jackson H, Hackett JT, Rubel EW (1982) Organization and development of brain stem auditory nuclei in the chick: ontogeny of postsynaptic responses. J Comp Neurol 210:80-86.

Jhaveri S, Morest DK (1982a) Sequential alterations of neuronal architecture in nucleus magnocellularis of the developing chicken: a Golgi study. Neuroscience 7:837-853.

Jhaveri S, Morest DK (1982b) Sequential alterations of neuronal architecture in nucleus magnocellularis of the developing chicken: an electron microscope study. Neuroscience 7:855-870.

Joshi I, Wang LY (2002) Developmental profiles of glutamate receptors and synaptic transmission at a single synapse in the mouse auditory brainstem. J Physiol (Lond) 540:861-873.

Kakizawa S, Yamasaki M, Watanabe M, Kano M (2000) Critical period for activity-dependent synapse elimination in developing cerebellum. J Neurosci 20:4954-4960.

Kim G, Kandler K (2003) Elimination and strengthening of glycinergic/ GABAergic connections during tonotopic map formation. Nat Neurosci 6:282-290.

Kumar SS, Bacci A, Kharazia V, Huguenard JR (2002) A developmental switch of AMPA receptor subunits in neocortical pyramidal neurons. J Neurosci 22:3005-3015.

Lawrence JJ, Trussell LO (2000) Long-term specification of AMPA receptor properties after synapse formation. J Neurosci 20:4864-4870.

Lichtman JW, Colman H (2000) Synapse elimination and indelible memory. Neuron 25:269-278.

Mishina M, Takai T, Imoto K, Noda M, Takahashi T, Numa S, Methfessel C, Sakmann B (1986) Molecular distinction between fetal and adult forms of muscle acetylcholine receptor. Nature 321:406-411.

Nicol MJ, Walmsley B (2002) Ultrastructural basis of synaptic transmission between endbulbs of Held and bushy cells in the rat cochlear nucleus. J Physiol (Lond) 539:713-723.

Otis TS, Wu YC, Trussell LO (1996) Delayed clearance of transmitter and the role of glutamate transporters at synapses with multiple release sites. J Neurosci 16:1634-1644.

Parks TN (1981) Morphology of axosomatic endings in an avian cochlear nucleus: nucleus magnocellularis of the chicken. J Comp Neurol 203:425-440.

Rabacchi S, Bailly Y, Delhaye-Bouchaud N, Mariani J (1992) Involvement of the $N$-methyl-D-aspartate (NMDA) receptor in synapse elimination during cerebellar development. Science 256:1823-1825.

Rich MM, Lichtman JW (1989) In vivo visualization of pre- and postsynap- 
tic changes during synapse elimination in reinnervated mouse muscle. J Neurosci 9:1781-1805.

Rodriguez-Contreras A, de Lange RP, Lucassen PJ, Borst JG (2006) Branching of calyceal afferents during postnatal development in the rat auditory brainstem. J Comp Neurol 496:214-228.

Rubel EW, Parks TN (1988) Organization and development of the avian brain-stem auditory system. In: Auditory function (Edelman GM, Cowan WM, Gall WE, eds), pp 3-92. New York: Wiley.

Saunders JC, Coles RB, Gates GR (1973) The development of auditory evoked responses in the cochlea and cochlear nuclei of the chick. Brain Res 63:59-74.

Sugden SG, Zirpel L, Dietrich CJ, Parks TN (2002) Development of the specialized AMPA receptors of auditory neurons. J Neurobiol 52:189-202.

Swanson GT, Kamboj SK, Cull-Candy SG (1997) Single-channel properties of recombinant AMPA receptors depend on RNA editing, splice variation, and subunit composition. J Neurosci 17:58-69.

Tang YZ, Carr CE (2004) Development of NMDA R1 expression in chicken auditory brainstem. Hear Res 191:79-89.
Taschenberger H, von Gersdorff H (2000) Fine-tuning an auditory synapse for speed and fidelity: developmental changes in presynaptic waveform, EPSC kinetics, and synaptic plasticity. J Neurosci 20:9162-9173.

Tovar KR, Westbrook GL (1999) The incorporation of NMDA receptors with a distinct subunit composition at nascent hippocampal synapses in vitro. J Neurosci 19:4180-4188.

Trussell LO (1999) Physiology of glutamatergic transmission at calyceal and endbulb synapses of the central auditory pathway. In: Ionotropic glutamate receptors in the CNS (Jonas P, Monyer H, eds), pp 399-418. Berlin: Springer.

Wu G, Malinow R, Cline HT (1996) Maturation of a central glutamatergic synapse. Science 274:972-976.

Zhang S, Trussell LO (1994) Voltage clamp analysis of excitatory synaptic transmission in the avian nucleus magnocellularis. J Physiol 480: $123-136$.

Zhou N, Parks TN (1992) Developmental changes in the effects of drugs acting at NMDA or non-NMDA receptors on synaptic transmission in the chick cochlear nucleus (nuc. magnocellularis). Brain Res Dev Brain Res 67:145-152. 